\title{
What future for chest pain observation units?
}

Patients with new or worsening chest pain suggesting acute coronary syndromes need treating as an emergency. Rapid assessment is crucial but difficult. Patients with acute chest pain now represent $20 \%$ or $30 \%$ of all emergency medical admissions, yet less than one third of these will have acute coronary syndromes (acute myocardial infarction or unstable angina). ${ }^{1}$ The majority thus do not have significant coronary heart disease. There are approximately 600000 unnecessary admissions each year in the UK. If adequately investigated, on the basis of rule out algorithms, admission could be avoided. However, up to $2 \%$ of acute myocardial infarctions are mistakenly sent home with perhaps a $25 \%$ case fatality rate and a high risk of medicolegal problems. ${ }^{2-4}$ Any effective scheme would potentially save substantial NHS resources and reduce stress for patients, carers and staff. ${ }^{1}$

It is important to emphasise the social context. This is a continuous spectrum ranging from patients who never present grading imperceptibly into patients who present as emergencies. The trigger for such presentation represents the final step in a complex pathway commencing with the patient's perception of symptoms, illness behaviour, family and social norms, advice from significant others, level of education, availability of primary care support, access to accident and emergency (A\&E) and response of A\&E staff. Although the incidence of coronary heart disease is declining, admissions for chest pain are increasing, reflecting a complex system change with higher expectations and greater reluctance by medical staff to discharge patients without a firm diagnosis.

Same day "one stop" diagnosis outpatient clinics can safely avoid admission in most non-emergency patients. Such services are now well established in Edinburgh, Glasgow, Harefield, Hillingdon, Southampton and London. Patients are referred by their GP. The history is established and a clinical examination, resting ECG and usually an exercise ECG are performed. Management algorithms may be implicit, or explicit and formalised. Patients are categorised into low risk (clinically significant coronary heart disease unlikely), intermediate risk (angina of effort likely), and high risk (possibility of unstable angina or even acute myocardial infarction). High risk patients are usually admitted. Low risk and intermediate risk patient are usually appropriately advised and reassured. Further investigations, angiography or trial of angina medication for instance, are arranged where necessary. ${ }^{1}$

In Edinburgh, 317 patients referred by their GPs with new or increasing chest pain were seen within 24 hours. Only $18 \%$ needed admission for acute coronary syndromes. Altogether $82 \%$ were sent home, including 30\% with stable coronary heart disease. The $49 \%$ with non-cardiac chest pain were immediately reassured. ${ }^{5}$ This emphasises that psychosomatic chest pain is common.

Results seem encouraging from other studies involving cohorts of 93 to 1001 patients and a mean age ranging from 50 to 58 years. ${ }^{6-9}$ Between $8 \%$ and $26 \%$ had acute coronary syndromes with admission rates of $12 \%-80 \%$. Thus, up to $88 \%$ of patients were discharged home. Acute myocardial infarction or death subsequently occurred in only $0 \%-0.5 \%$ of discharged patients.

Such clinics have now received massive political and financial support from the National Service Framework for coronary heart disease. ${ }^{10}$ However, the existing data describing rapid access chest pain clinics have major limitations. All were cohort studies with variable inclusion cri- teria and losses to follow up. Big selection biases are therefore possible. Admissions might have been halved, ${ }^{11}$ but true effectiveness may have been much less. Politicians, civil servants and some health authorities are already convinced about their effectiveness. Sceptics are concerned about potential increases in patient numbers, referrals for angiography and revascularisation and hence substantial NHS costs. Important questions remain: the proportion of patients needing revascularisation and actually getting it, whether medical treatment is optimised first, whether symptoms are generally improved and both the effectiveness and cost effectiveness of one stop clinics.

Unlike "one stop" clinics, chest pain observation units (CPOUs) generally target patients presenting as an emergency. Most CPOUs provide observation for six hours or more, commencing with history, examination, resting ECG and baseline cardiac enzyme activities. Having established a low probability of infarction or unstable angina an exercise ECG is often performed. Patients can then be rapidly categorised into high or medium risk requiring admission, and the majority into low risk who can be safely discharged because significant coronary heart disease is unlikely. Patient management is generally based on a myocardial infarction rule out algorithm (ROMI). Extensive CPOU experience is now reported in the United States ${ }^{11}{ }^{12}$ and in British practice in Manchester and Sheffield. ${ }^{13}{ }^{14}$ Some further experience has been reported from Barnsley, Greenwich, Leeds, Southampton Whiston (Goodacre SW, personal communication) and Bath. ${ }^{15}$ CPOUs therefore seem safe and effective in British practice studies. Impressively up to $80 \%$ of patients can be discharged safely compared with only about $33 \%$ in most acute hospitals.

In this month's journal, Herren and Mackway-Jones (page 6) review the evidence base underlying diagnostic and treatment strategies for patients with cardiac sounding chest pain. They conclude that CPOUs are the best way to implement rapid, cost effective and efficacious ROMI protocols so that low risk patients are discharged safely. To rule out myocardial infarction in patients with fewer than 12 hours of chest pain, a combination of CK-MB measurements and ST segment monitoring has been shown to be safe when compared with normal inpatient care. However, they suggest that important questions remain about the efficacy of other test combinations, such as the troponin tests with exercise tests and echocardiograms, for $\mathrm{A} \& \mathrm{E}$ patients.

In the United States, mean cost savings of around $\$ 560 / \$ 650$ per patient have been reported in RCTs, and up to $\$ 2000$ in cohorts. American RCT data are however limited. "Rapid" assessment could take up to 50 hours and all studies were small and powered to detect differences in costs not outcomes. Savings were demonstrated in comparison to high cost USA inpatient controls. Circumstances in the British NHS might be rather different.

Goodacre and colleagues (page 11) report on whether a CPOU is likely to be cost saving in a British hospital. They warn that cost savings demonstrated in US studies should not be extrapolated to the UK context. Furthermore, including interventional cardiology costs in an economic evaluation is recommended as an important determinant of cost effectiveness. Savings might only amount to $£ 150$ or $£ 200$ per patient. ${ }^{13}$ They conclude that economic evaluation of the CPOU should therefore be repeated in the UK. 
In conclusion, unbiased British data from a RCT are now therefore essential to measure efficacy and cost effectiveness. This RCT would aim to recruit the majority of acute patients seen every day. Funding from a large charity is being actively sought and centres wishing to collaborate in a multicentre RCT would be welcome.

SIMON CAPEWELL

DEBORAH QUINNEY

Department of Public Health, University of Liverpool, Whelan Bldg Quadrangle, Liverpool L69 3GB, UK

Correspondence to: Professor Capewell (capewell@liverpool.ac.uk)

1 Capewell S, McMurray JJV. "Chest Pain-Please Admit", is there an alternative? $B M \mathcal{F}$ 2000;320:951-2.

2 Mehta RH, Eagle KA. Missed diagnoses of acute coronary syndromes in the emergency room - continuing challenges. NEFM 2000;342:1207-10.

3 Collinson PO, Premachandram S, Hashemi K. Prospective audit of incidence of prognostically important myocardial damage in patients discharged from emergency department. $B M \mathcal{F} 2000 ; 320: 1702-5$

4 Kennedy RL. Commentary: Time for improved diagnosis and management of patients presenting with acute chest pain. BMF 2000;320:1704-5.

5 Davie AP, Caesar D, Caruana L, et al. Outcome from a rapid assessment chest pain clinic: closing Pandora's box? QF Med 1998;91:339-43.
6 Newby DE, Fox KAA, Flint LL, et al. A 'same day' direct-access chest pain clinic: improved management and reduced hospitalization. Qf Med 1998; 91:333-7.

7 Norell M, Lythall D, Coghlan G, et al. Limited value of the resting electrocardiogram in assessing patients with recent onset chest pain: lessons from a chest pain clinic. Br Heart f 1992;67:53-6.

8 Roberts RH, McEvoy C, Stock K, et al. The incidence and presentation of ischaemic heart disease: a population survey. Br Heart 7 1995;73 (suppl 3): 49 .

9 Ghandi MM, Lampe FC, Wood DA. Incidence, clinical characteristics and short term prognosis of angina pectoris. Br Heart F 1995;73:193-8.

10 National Service Framework for coronary heart disease. London: Department of Health, 2000.

11 Goodacre SW. Should we establish chest pain observation units $\mathrm{n}$ the United Kingdom? A systematic review and critical appraisal of the literature. 7 Accid Emerg Med 2000;17:1-6.

12 Quin G. Chest pain evaluation units. F Accid Emerg Med 2000;17:237-40.

13 Goodacre SW, Morris FP, Campbell S, et al. A descriptive study of a chest pain observation unit in a UK hospital. [Abstract]. F Accid Emerg Med 2000;17:58.

14 Richell Herren KR, Mackway-Jones K, et al. Early recognition of myocardial damage: an emergency department based diagnostic test study. [Abstract]. f Accid Emerg Med 2000;17:59.

15 Meek S. Chest pain rapid rule out is already here. Electronic response to prospective audit of incidence of prognostically important myocardial damage in patients discharged from emergency department. bmj.com 2000;320 (www.bmj.com/cgi/eletters/320/7251/\#EL1; accessed 30 July.) 